\title{
Measurement of dissolved gases in anaerobic biogenic media
}

\author{
M. Schelter, J. Zosel, F. Berthold, W. Oelßner, U. Guth \\ Meinsberg Kurt-Schwabe Research Institute \\ Kurt-Schwabe-Straße 4, \\ D-04720 Ziegra-Knobelsdorf, Germany
}

\section{Outline of the problem}

The anaerobic production of biogas from biomass is one of the most important pillars of renewable energy sources worldwide. This transformation, starting with the hydrolysis of the input material and ending up after several intermediate steps with the methanogenesis [1], is based on the complex teamwork of thousands of different microbes. Therefore, the parameter window of optimum process conditions is relatively narrow and has to be controlled carefully. The concentration of dissolved hydrogen is one of the key parameters known to indicate process stability limitations [2]. In this work a measuring system for dissolved hydrogen in anaerobic biogenic media with high selectivity and sensitivity is presented, which provides high long-term stability and maintenance free operation.

\section{Innovation of solution}

Due to the fact that a long-term stable and selective measurement of dissolved hydrogen with membrane covered amperometric sensors is connected with some inevitable difficulties [3], a new approach was taken into account, which is based on a membrane free extraction of small amounts of dissolved substances by a carrier gas flow. After chromatographic separation the components of this gas mixture are measured in a high temperature solid electrolyte cell made of yttria stabilized zirconia (YSZ). The cell is operated in a coulometric regime, resulting in the immediate total titration of hydrogen and other reducing gases by oxygen ions pumped through the YSZ. This method enables highly sensitive and calibration-free measurements without baseline drifting. The limit of detection is lower than 1 vol.-ppm $\mathrm{H}_{2}$.

\section{Technical realisation}

The newly developed system, given in figure 1, contains an extractor, a gas chromatograph and an YSZ cell acting as detector. An oxygen free gas fed into the extractor at $15 \mathrm{~mL} / \mathrm{min}$ contacts the biogas medium at the membrane free boundary below the extractor causing the permeation of defined amounts of dissolved gases. This mixture is forwarded then to the sample loop of the gas chromatograph. A column packed with molecular sieve provides the separation of hydrogen and methane within 10 min at $50{ }^{\circ} \mathrm{C}$ column temperature in argon carrier gas flow at $20 \mathrm{~mL} / \mathrm{min}$. Situated at the column end the coulometric detector $\mathrm{YSZ}$ cell is operated at $750^{\circ} \mathrm{C}$.

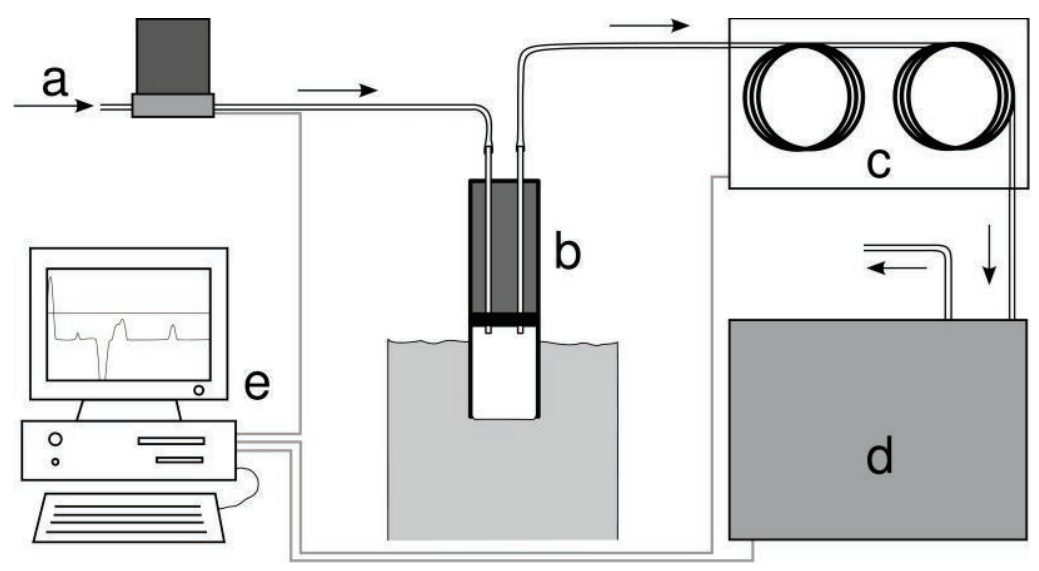

Fig. 1:

Principle of dissolved gas measurement,

a extraction gas supply with mass flow control,

b extractor providing interface between extraction gas and aqueous medium,

c gas chromatograph with silica gel and molecular sieve column,

d coulometric detector with YSZ cell,

e computer for control and evaluation

\section{New results}

The curve in figure 2 shows linear response of the coulometric detector to hydrogen concentrations between $1 \ldots 100$ vol.-ppm. The slope of the curve is equal to that calculated by Faraday's law. A small offset comes with residual oxygen in the carrier gas. 


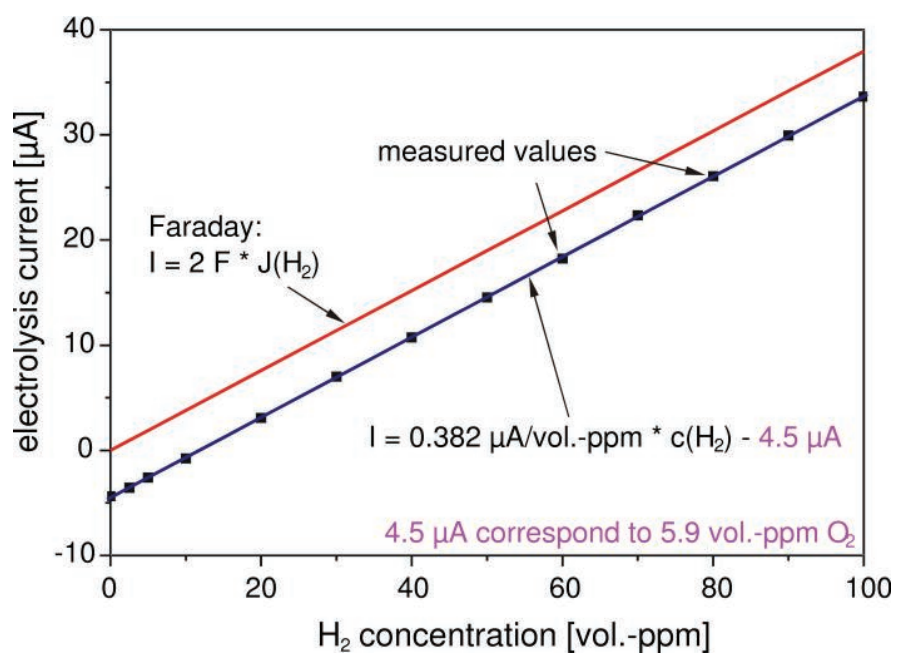

Fig. 2:

Electrolysis current through the coulometric detector cell, comparison between the measured values and the current necessary for complete titration of hydrogen, calculated by Faraday's law, carrier gas $\mathrm{N}_{2}$ at $2.6 \mathrm{~mL} / \mathrm{min}$

The complete measuring system consisting of extractor, gas chromatograph and coulometric detector was investigated over three days in distilled water with defined amounts of dissolved hydrogen and methane as shown in figure 3. The curves indicate constant transfer of both gases into the extraction gas flow as well as long-term stable performance of the chromatographic measurement.

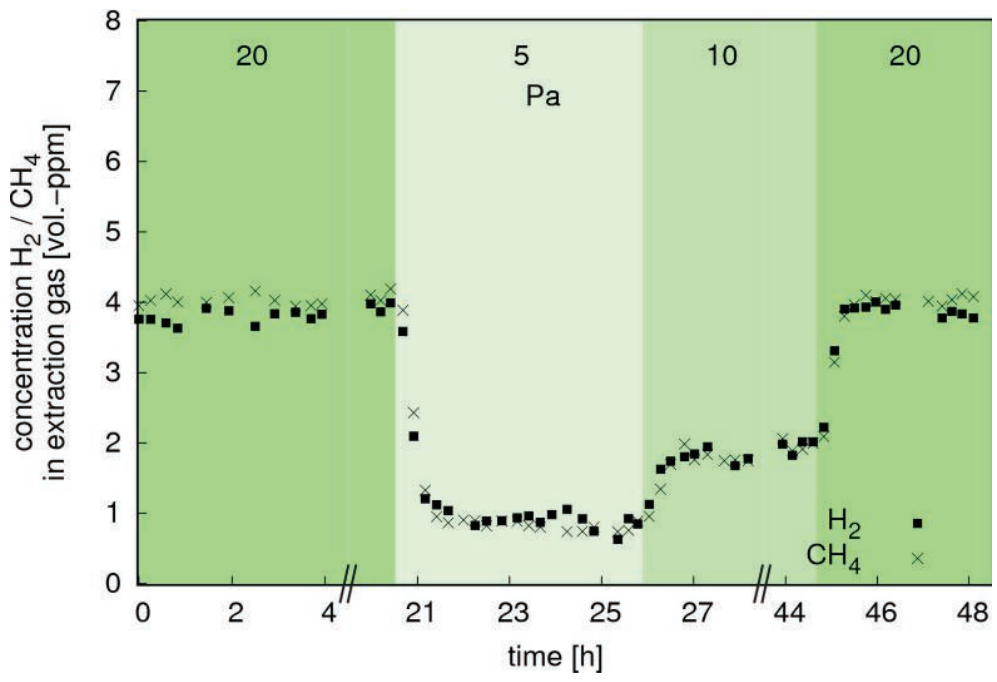

Fig. 3:

Concentrations of hydrogen and methane measured in the extracted gas over 48 hours at different dissolved gas partial pressures in distilled water (values at top of graph) at $25^{\circ} \mathrm{C}$, extraction gas $\mathrm{N}_{2}$ with flow rate $2 \mathrm{~mL} / \mathrm{min}$

Furthermore, the measuring system was tested on sensitivity, temperature dependence and extraction gas flow rate with different extractor geometries.

Examined twice in a biogas lab plant, the measuring system delivered chromatograms over two days with manual operation and over fourteen days operating automatically. The extractor plunged about $5 \mathrm{~cm}$ into the fermentation medium while nitrogen as extraction gas was pumped through it.

Shown in figure 4 , the collected partial pressure courses of hydrogen and methane during the first measuring campaign prove fast response to concentration changes. After feeding the fermenter with maize silage both partial pressures increased to the three- to fourfold and went back to starting values within three hours.

Figures 5 to 7 show the results of the second measuring campaign in the biogas lab plant. The course of extracted amounts of hydrogen in comparison to simultaneously taken hydrogen concentrations in biogas are displayed in figure 5. The basis concentration of dissolved hydrogen increases with the amount of added feed, highlighted by the thickness of black arrows. This effect cannot be seen in the hydrogen concentration in biogas, so the measurement of dissolved hydrogen is much more sensitive and therefore it is a much better indicator for the stability of the fermentation process. The detail of a feeding peak 
shown in figure 6 points out an ultrafast response of dissolved hydrogen after feeding the reactor, whereas the hydrogen concentration peak in the biogas is delayed by one hour.

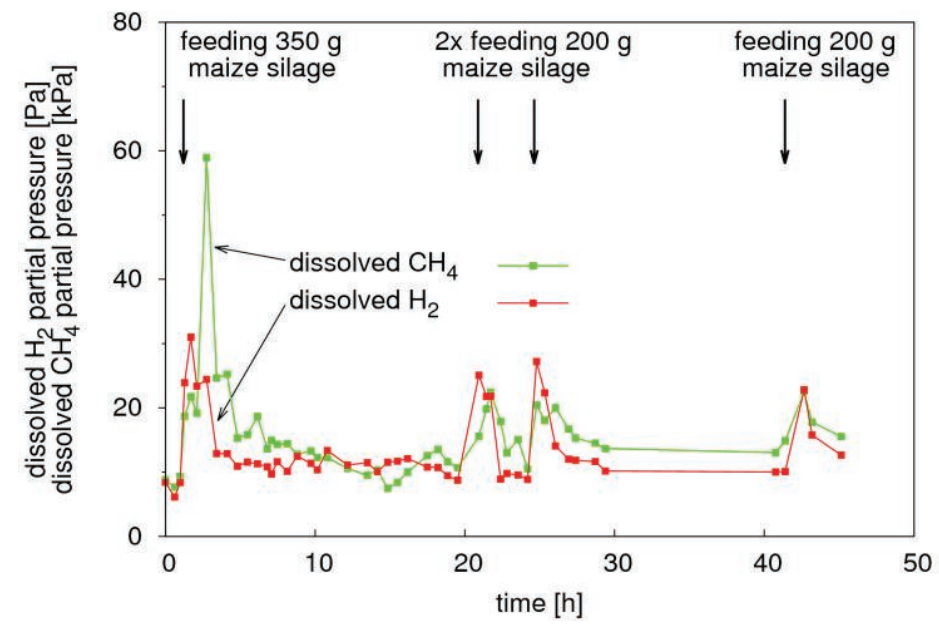

Fig. 4:

Results of the first measuring campaign in a biogas lab plant

Methane was also extracted and quantified as shown in the results in figure 7 . Similar to hydrogen, it can be figured out an increasing basis concentration of dissolved methane with growing feeding amounts and also a much faster increasing of dissolved methane concentration compared to the biogas concentration.
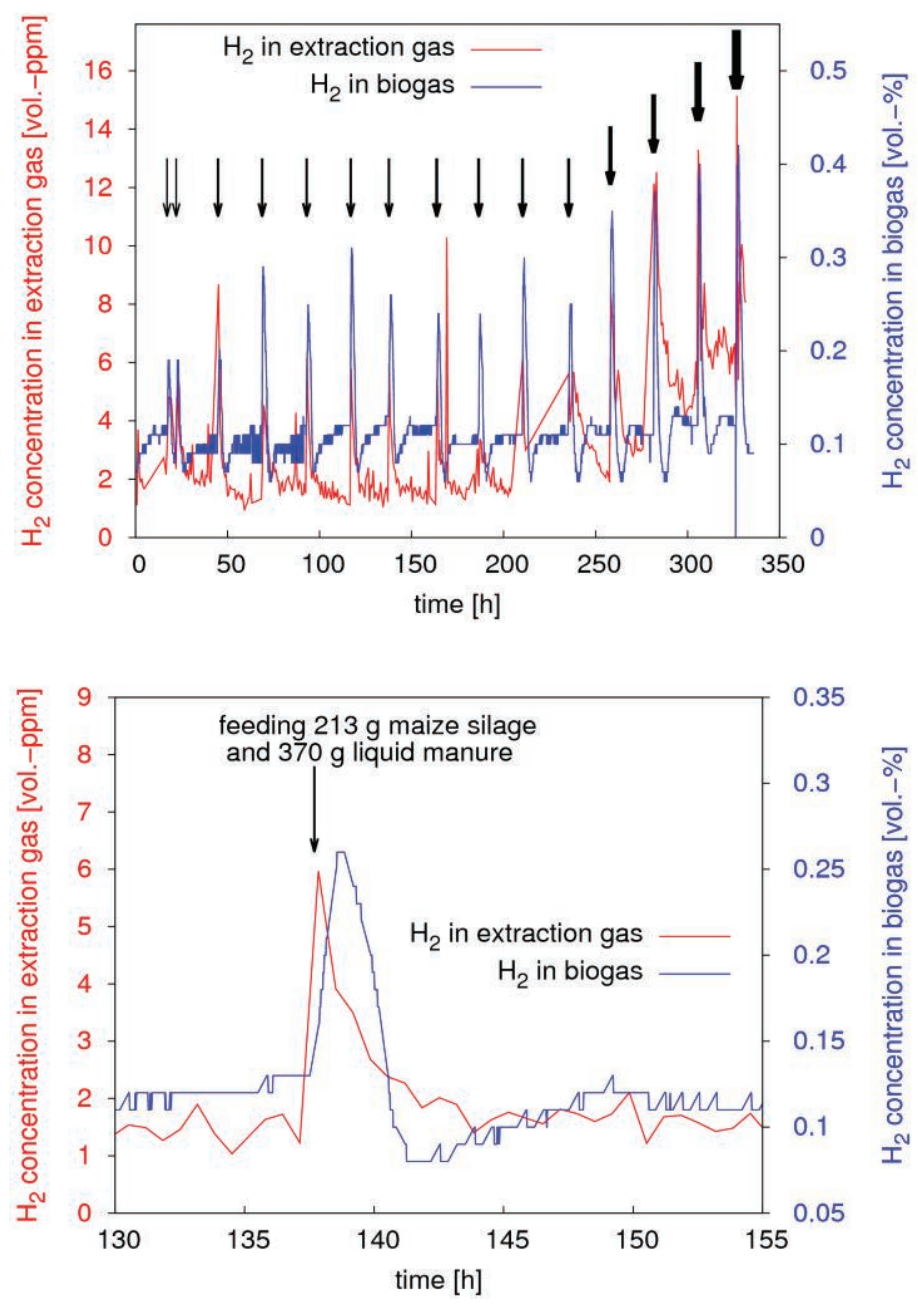

Fig. 5:

Results of both dissolved hydrogen and hydrogen in biogas during the second measuring campaign in a biogas lab plant, black arrows pointing to moments of feeding the fermenter with maize silage and liquid manure whereas the arrow thickness is proportional to the amount fed
Fig. 6:

Detail of figure 5, the arrow points to the exact feeding time 


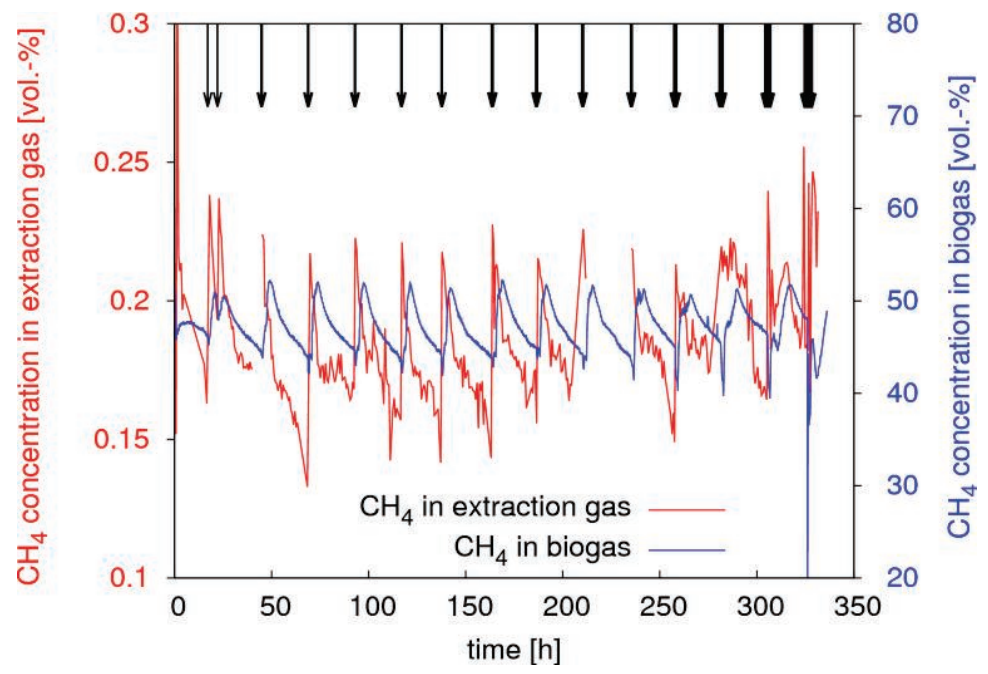

Fig. 7:

Results of both dissolved methane and methane in biogas during the second measuring campaign in a biogas lab plant, black arrows pointing to moments of feeding similar to figure 5

A new extractor developed for usage in a biogas plant was introduced through a nozzle in a digester as pictured in figure 8. During the measurements the digester was fed with maize silage, cow dung and liquid manure. All components besides the extractor were placed in a container shown in figure 9. Operated manually, the measuring system delivered chromatograms every 20 minutes over 50 hours.

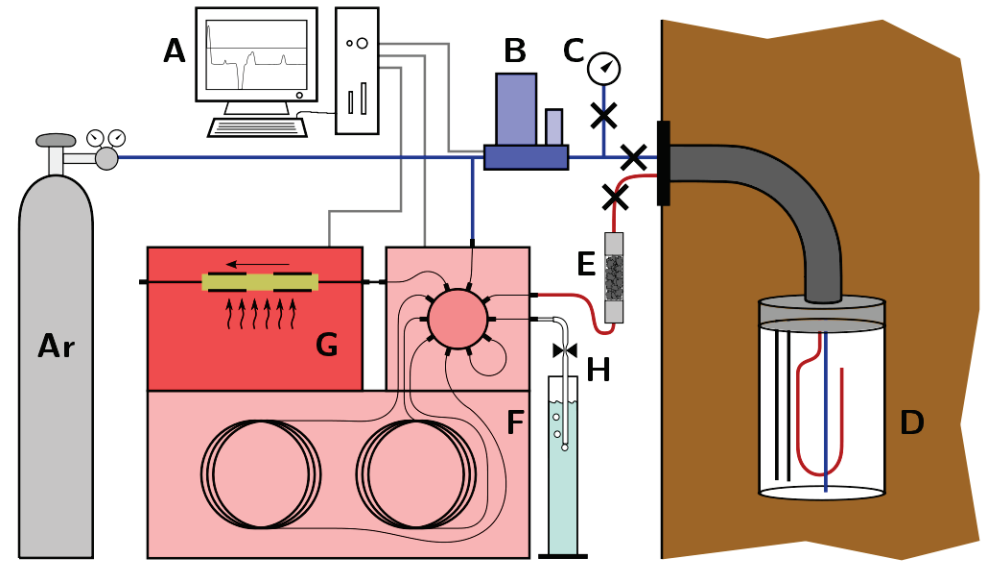

Fig. 8:

Measuring system for dissolved gas analysis in a biogas plant:

A control unit,

B mass flow controller,

C manometer,

D extractor in fermentation medium,

E $\mathrm{H}_{2} \mathrm{~S}$-filter,

$\boldsymbol{F}$ gas chromatograph,

$\boldsymbol{G}$ coulometric detector,

$\boldsymbol{H}$ counter-pressure adjustment, argon as extraction gas and carrier gas for the gas chromatograph

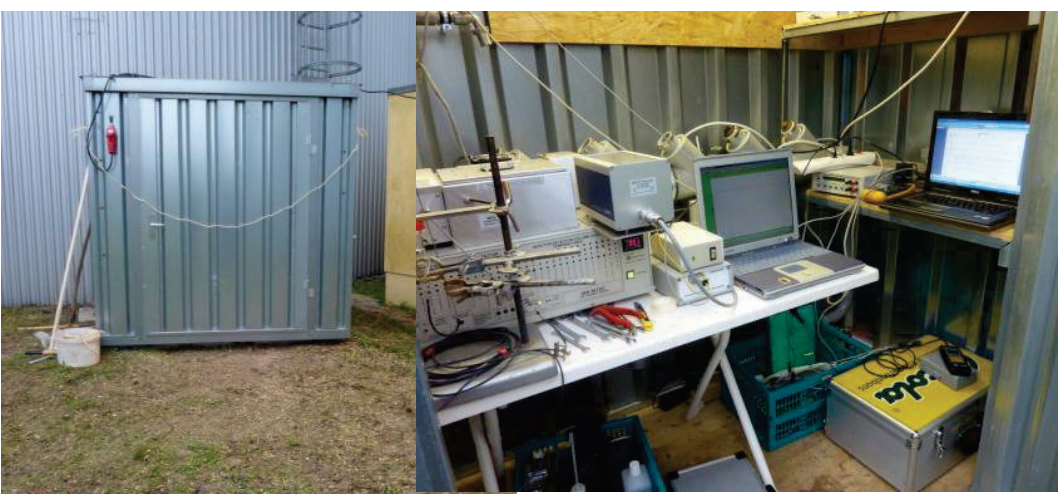

Fig. 9:

Container with measuring

system in front of the fermenter

Figure 10 shows the progress of the concentrations of methane, oxygen and hydrogen in the extraction gas. The obtained methane concentrations fluctuate over several orders of magnitude showing that the methane formation potential of the digester sludge near the extractor varies in a wide range.

The basis hydrogen concentration in the extraction gas is about $1 \ldots 2.5$ vol.-ppm while single peaks of up to 6 vol.-ppm arise especially after the feeding of cow dung. At the same time there is no increasing 
hydrogen amount in biogas, whereas it was observed months before after feeding large amounts of cow dung. Therefore this is another evidence of the high sensitivity of the described dissolved gas analysis system and also the proof-of-principle of long-term stable on-site measurement of hydrogen in biogas.

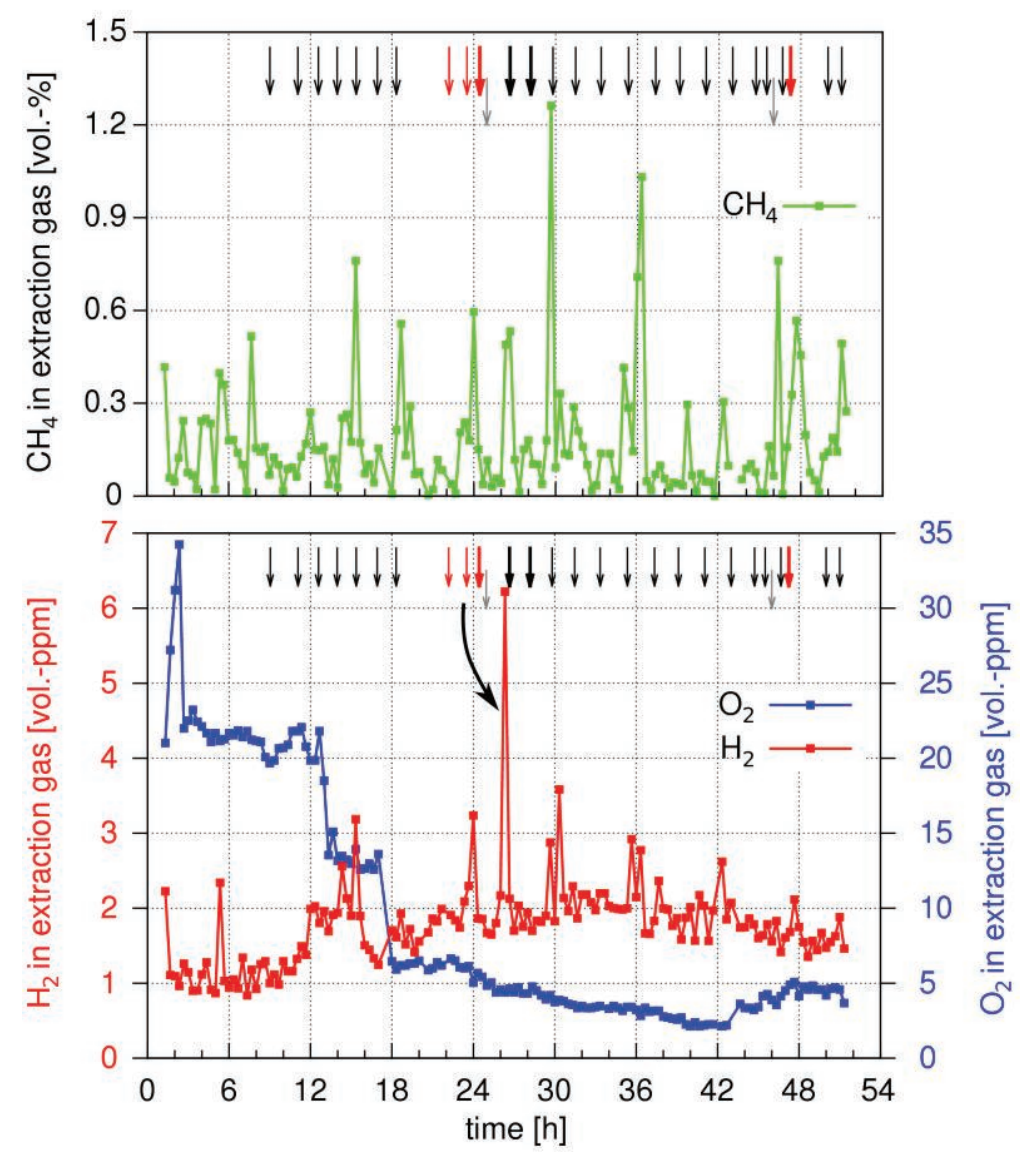

Fig. 10:

Results of dissolved gas analysis in a biogas plant, black arrows: feeding of maize silage, red arrows: feeding of maize silage and cow dung, grey arrows: feeding of liquid manure

\section{Conclusions}

A measuring system was developed to measure low concentrations of dissolved gases in anaerobic biogenic media by coulometric detection after extraction and chromatographic separation. Tests under real conditions proof long-term stable performance with high sensitivity and selectivity. Testing the system in a biogas lab plant and a biogas plant proves the stable performance and the reliable resolution of dissolved hydrogen with high selectivity.

\section{Literature}

[1] D. Deublein, A. Steinhauser: Biogas from Waste and Renewable Resources. Wiley-VCH, Weinheim, 2008.

[2] A. Pauss et al.; Appl. Environ. Microb. 56 (1990), 1636-1644.

[3] J. Zosel, et al.; Chemie Ingenieur Technik 79 (2007), 1339.

\section{Acknowledgement}

The support of the Federal Ministry of Food, Agriculture and Consumer Protection is gratefully acknowledged (support code 22009407). 